DOE/CE/26602-3

\title{
DEVELOPMENT OF \\ ADVANCED LOW-TEMPERATURE HEAT TRANSFER FLUIDS \\ FOR DISTRICT HEATING AND COOLING
}

Progress Report

for Period March 31, 1991-June 30, 1991

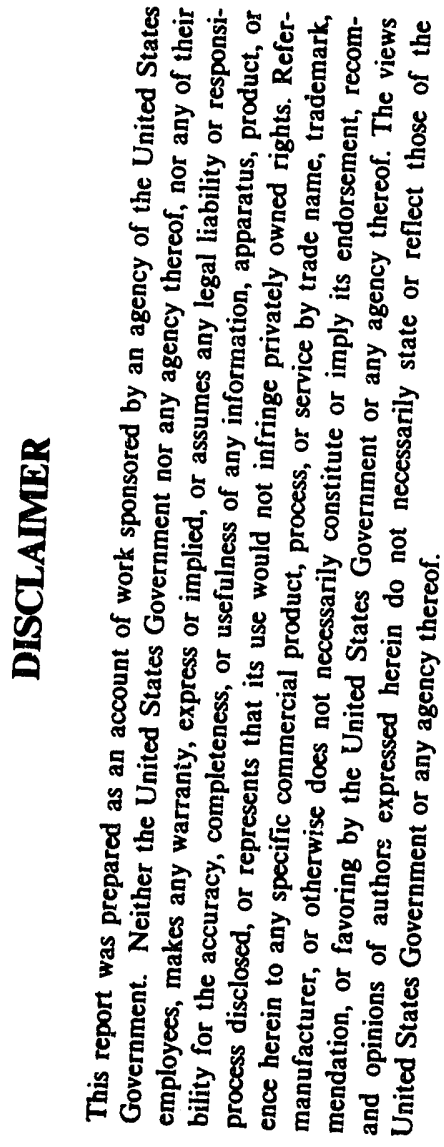

Drexel University

Philadelphia, Pennsylvania 19104

June 30, 1991

Prepared for

THE U.S. DEPARTMENT OF ENERGY

AGREEMENT NO. DE-FG01-90CE26602 


\title{
EFFECTS OF EMULSIFIER ON PARTICLE SIZE OF A PHASE CHANGE MATERIAL IN A MIXTURE WITH WATER
}

\author{
Eunsoo Choi, Young I. Cho, and Harold G. Lorsch \\ Department of Mechanical Engineering and Mechanics \\ Drexel University, Philadelphia, PA 19104
}

\begin{abstract}
The use of a phase-change material (PCM) in a district cooling system was studied in order to increase the heat transfer performance of the system. One of the major technical obstacles to its use is the clogging of the piping system. The present study attemps to minimize the clogging problem by using an emulsifier. Effects of the emulsifier on the mixture of water and hexadecane (a PCM) were studied. A small amount (60 to 2,775 ppm) of emulsifier caused remarkable effects resulting in the production of fine PCM particles. As the amount of the emulsifier was increased in the $25 \%$ hexadecane mixture with water, the size of the solid particles of hexadecane became smaller. When the size of the hexadecane particles was small enough, they did not stick together or stick to the surface of the cold wall of a heat exchanger.
\end{abstract}

\section{Introduction}

Chilled water is used as the heat transfer fluid in district cooling systems. Recently, an ice slurry system has been investigated to increase the heat capacity of the fluid [1,2]. Chilled water can remove $20-40 \mathrm{MJ} / \mathrm{m}^{3}$ for an operating temperature range of $5-10^{\circ} \mathrm{C}$, but a $25 \%$ solid ice-slurry can remove $80 \mathrm{MJ} / \mathrm{m}^{3}$. If the ice slurry system can be successfully developed, the heat capacity of the working fluid will be increased 2-4 times, and the size of the district cooling machinery can be reduced accordingly. When the temperature of the working fluid is too low (i.e., near zero degree Celsius), condensation of ice particles on the surface of the heat exchanger will be severe, and the thermodynamic efficiency will be decreased. Hence, the recommended temperature of the heat transfer fluid for a district cooling system is approximately $10^{\circ} \mathrm{C}$. Another important problem in the use of ice-slurry systems is the clogging of the piping system. When ice particles are slightly sticky, which occurs during melting, they can stick together to form large lumps. Furthermore, such sticky ice particles or large lumps tend to stick to surfaces of the heat exchanger, eventually 
clogging the piping system. Thus, it is a major technical challenge to produce non-sticky fine ice particles when one tries to use ice-slurry systems.

Alternatively, mixtures of chilled water and n-alkane phase-change materials have been tested as a potential working fluid in a simulated district cooling system [3]. The n-alkane consists of simple, long molecular chains of $\mathrm{CH}_{2}$ units with a $\mathrm{CH}_{3}$ unit at each end. Its melting temperature depends on the length of the chain, increasing with increasing chain length. If n-alkane solid particles are used instead of ice particles, it may be possible to optimize the temperature of the working fluid to minimize the condensation problem. The fusion energies of the pure (100\%) nalkane PCMs are lower than that of water but fairly high (about $180 \mathrm{MJ} / \mathrm{m}^{3}$ ). In order to make it possible to use a mixture of water and n-alkane as the working fluid in a district cooling system, a proper method to generate non-sticking fine PCM particles of n-alkanes must be developed. The idea of using microencapsulated phase change material was proposed recently, but no experimental data exist yet [4]. The objective of the present investigation was to study the possibility of using an emulsifier to generate non-sticky fine PCM particles and to demonstrate the feasibility of generating such particles in a simulated heat exchanger experiment. The emulsifier used in the present study consisted of linear alkylbenzene sulfonate, poiyethoxylated fatty alcohol, and triethanolamine.

\section{Direct Contact Heat Exchange Experiments}

In the mixture of water and n-alkane the two materials are immiscible. The molecules of an emulsifier are cornposed of a hydrophilic head group and hydrophobic tail. When one applies a very small amount of emulsifier to the mixture of water and n-alkane, the n-alkane becomes the "internal" or "dispersed" phase, and water becomes the "external" or "continuous" phase.

At first, the effect of the amount of emulsifier on the mixture of water and hexadecane in a simple mixing procedure was studied. In Fig. 1, the experimental method is briefly explained. In Fig. 1(a), $5 \mathrm{cc}$ of distilled water was placed in a $40 \mathrm{cc}$ sample bottle, and a small amount of the emulsifier was added. In Fig. 1(b), $5 \mathrm{cc}$ of a commercial-grade hexadecane was added. The density of hexadecane was smaller than that of water, so that all the added hexadecane floated on the top of the water. When the bottle was gently shaken, an emulsion of water and hexadecane was formed as shown in Fig. 1(c). Since the emulsifier tends to increase the interfacial area of water and oilic substance, and since the interfacial area of small particles is larger than that of larje particles, the size of the liquid particles of hexadecane became smaller as the amount of emulsifier was increased. In Fig. 1(d), $10 \mathrm{cc}$ of cold water (at $4^{\circ} \mathrm{C}$ ) was injected using a syringe, and the bottle was shaken again. The emulsified liquid particles of hexadecane became frozen and suspended.

A Nicon F3 camera and micro lens were used to take pictures of the solidified particles. The volume percentage of the hexadecane in water was $25 \%$. Note that in the suspension flow, the upper limit of a dilute suspension is approximately $25 \%$. If the fraction of the suspended particles 


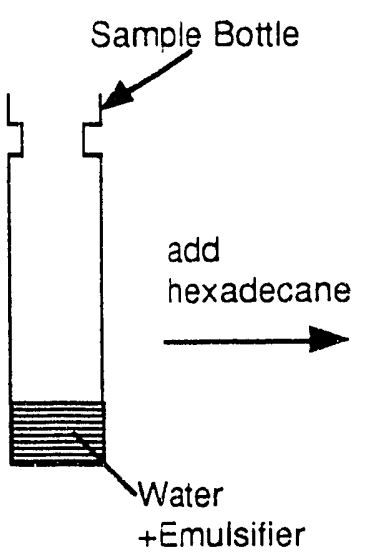

(a)

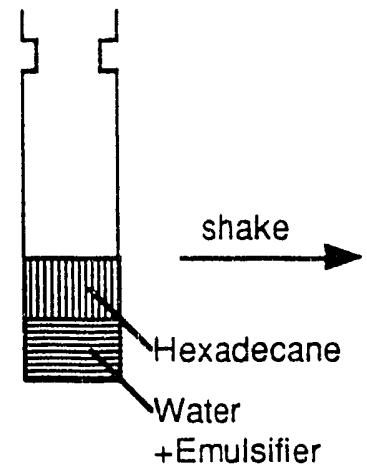

(b)

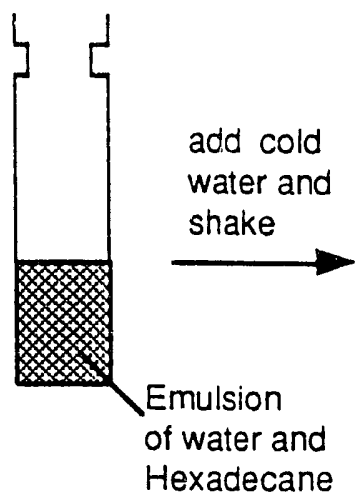

(c)

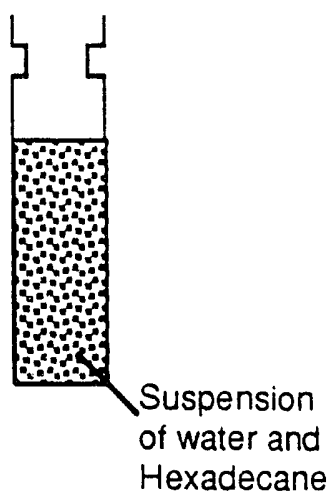

(d)

FIG 1

Procedure of making suspension from the mixture of water and hexadecane

is larger than $25 \%$, the property of the flow becomes very complex. The above experiment was repeated with different amounts of the emulsifier.

\section{Simulated Heat Exchanger Experiments}

In order to examine the above result in a realistic flow loop application, a simulated heat exchanger test was constructed generating PCM particles as shown in Fig. 2. A 25/75\% mixture by volume of hexadecane and water with a fixed amount of emulsifier was placed in a thermally insulated bath. A stirrer was used to make an emulsion of the mixture. A heat exchanger made of a copper tubing was inserted in the bath. From a constant-temperature circulating bath, cold water at $4^{\circ} \mathrm{C}$ was circulated through the heat exchanger. The temperature of the mixture decreased gradually, and the solidification of hexadecane particles was carefully observed. This test was repeated with different amounts of emulsifier.

\section{Results and Discussion}

From the direct contact heat exchange experiments, the significant effect of the emulsifier on the solidification of hexadecane particles could be observed. Fig. 3 shows the photographs of solidified hexadecane particles when they were spread on a petri dish. A centimeter scale is shown on the right hand side of each picture. When the emulsifier was not used, irregular, large lumps of solid hexadecane particles were formed as soon as the cold water was added as shown in Fig. 3(a). The solidification was observed to be initiated at a number of locations, and small particles, once formed, quickly grew into large lumps. These lumps were very stiff and difficult to break up, suggesting that, if these lumps are present in a flow loop, they will clog the loop immediately. In Fig. 3(b), 29 ppm of emulsifier was added to the water-hexadecane mixture, and smaller lumps of 


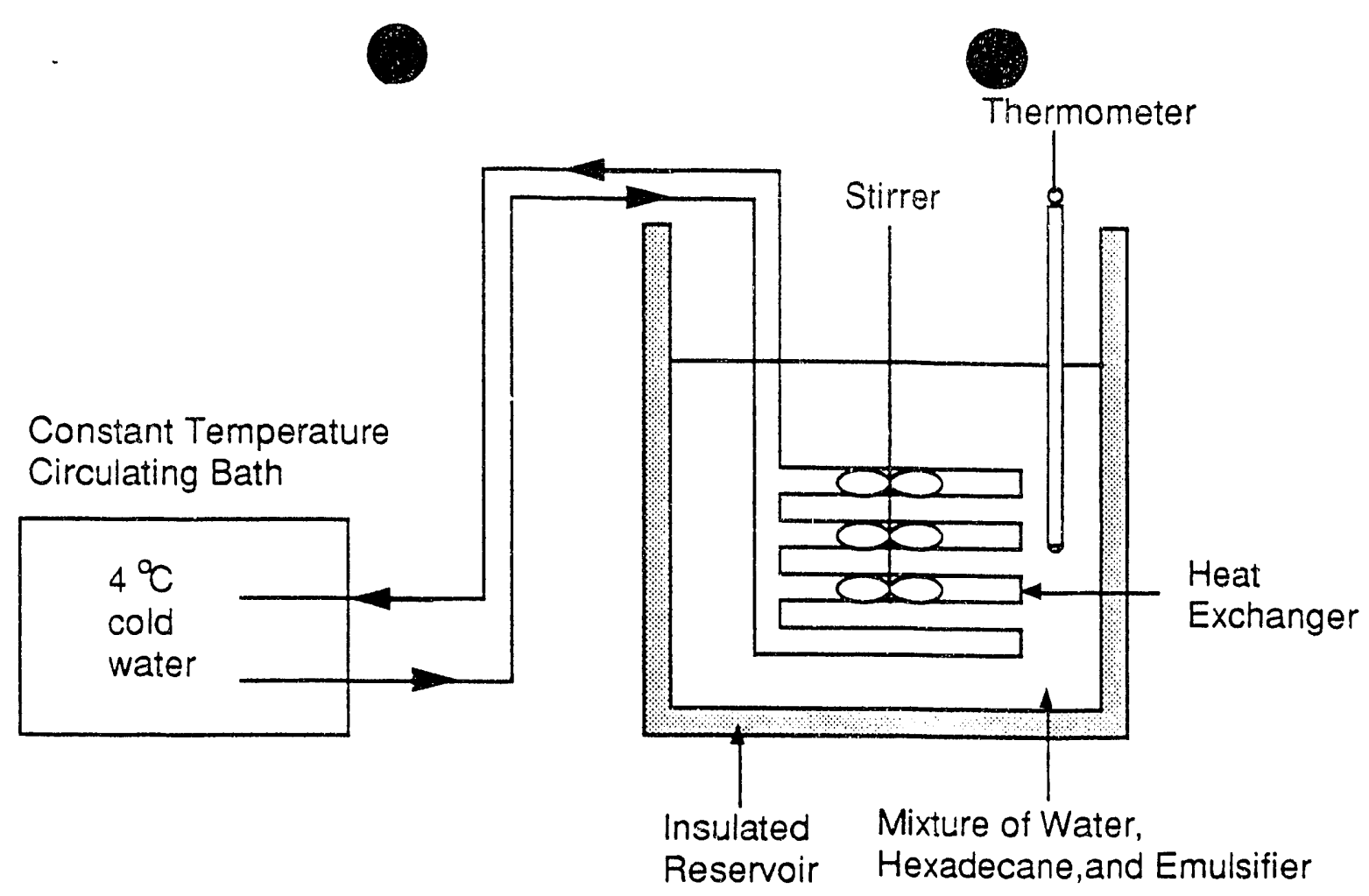

FIG 2

Schematic diagram of the cooling e perimental system using a heat exchanger

solid hexadecane particles were formed. The remarkable effect of adding more emulsifier is shown in Fig. 3(c). The size of the hexadecane particles was uniform and small. As the amount of emulsifier was increased, the size of the solid hexadecane particles became smaller as depicted in Figs. 3(b) to (g). The diameter of the particles shown in Fig. 3(d) was smaller than $1 \mathrm{~mm}$. There were no lumps at all with an amount of emulsifier of $680 \mathrm{ppm}$ or greater as demonstrated in Figs. 3(e) and (f). The size of the solid particles became too small to be seen by the naked eye in Fig. 3(g). Solid plastic spheres of $0.4 \mathrm{~mm}$ diameter are shown in Fig. 3(h) for comparison.

When the mixture solution was agitated with a stirrer, the solid hexadecane particles collided. When the solid hexadecane particles were large, they could stick together. Hence, it appears that there is a critical particle size below which the hexadecane particles cannot stick together. When the size of the particles is large, it is speculated that the inertial force of each particle is so large that it breaks the emulsified layer on the surface of the particle. Subsequently, the surface of the particle makes direct contact with other particles to form large lumps. When the size of a particle is small, the inertial force of the particle is so small that it is repelled by the electric charge on the emulsified layer. In this case, the emulsified liquid particles of hexadecane are frozen to become suspended solid particles without sticking to each other.

It can therefore be concluded that when one cools an emulsion of water and n-alkane to a temperature lower than the freezing temperature of the n-alkane, one can produce very small and 


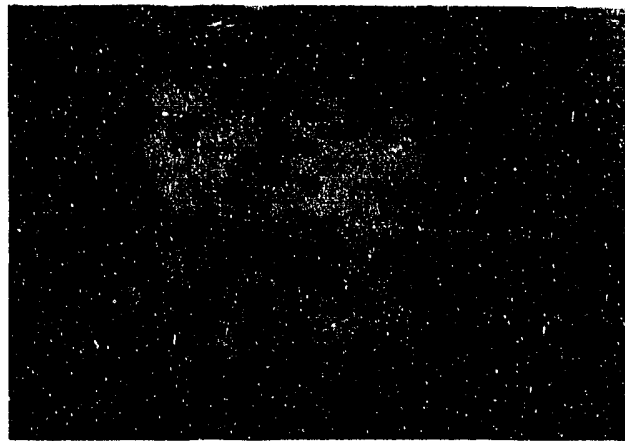

(a)

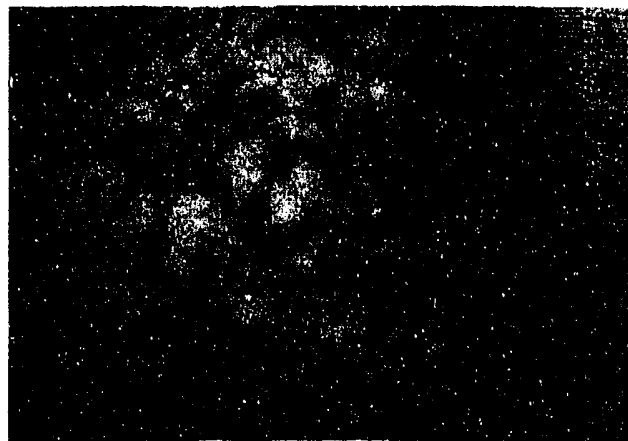

(b)

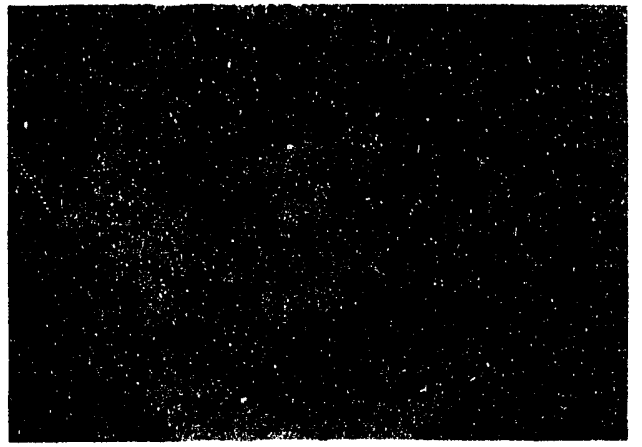

(c)

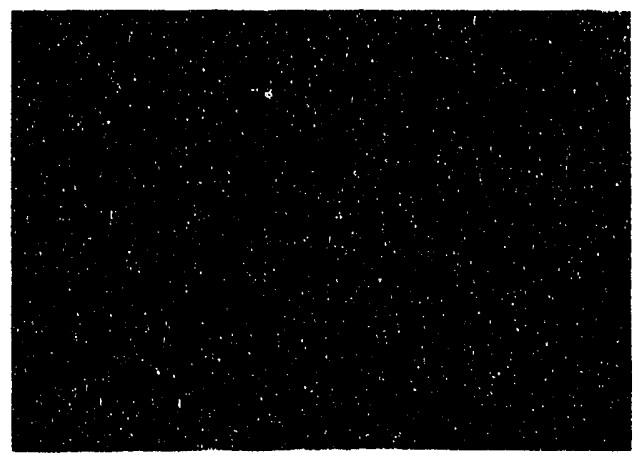

(d)

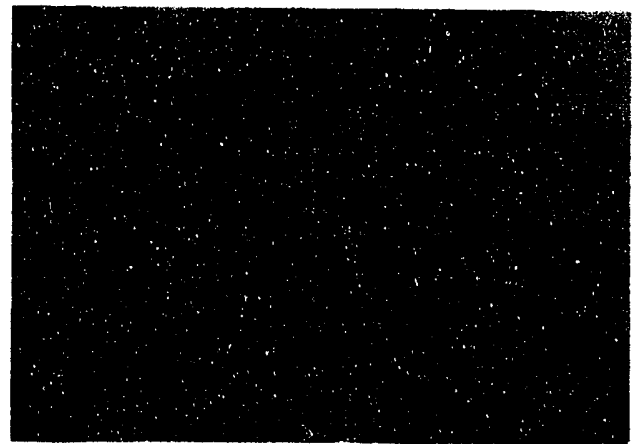

(e)

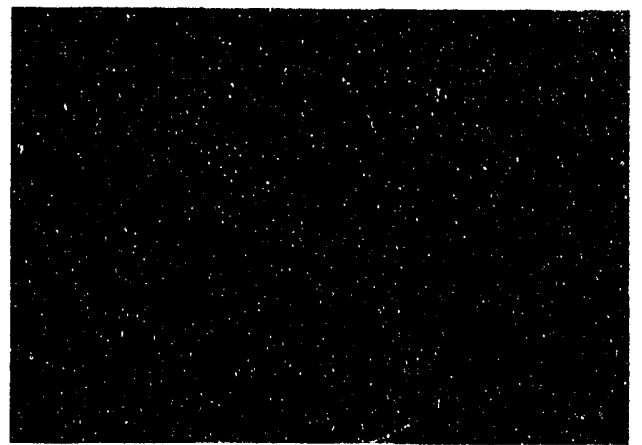

(f)

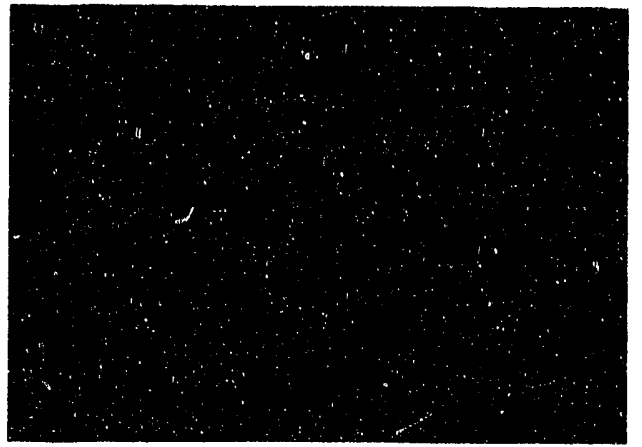

(g)

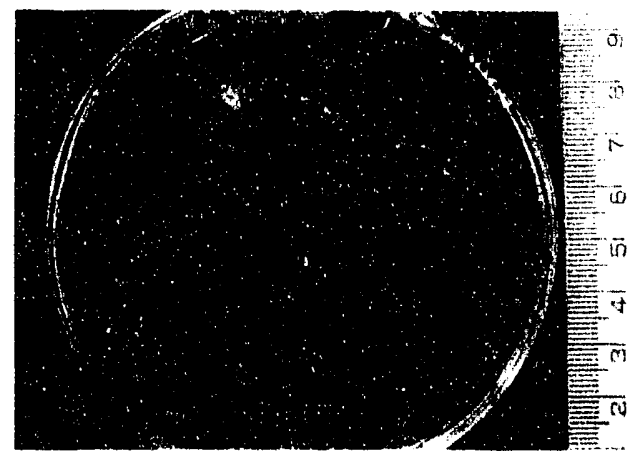

(h)

FIG 3

Photographs of solidified hexadecane particles produced in the direct contact heat exchange experiment with various amounts of emulsifier in a $25 / 75 \%$ hexadecane-water mixture : (a) () $\mathrm{ppm}$, (b) $29 \mathrm{ppm}$, (c) $60 \mathrm{ppm}$, (d) $273 \mathrm{ppm}$, (e) $680 \mathrm{ppm}$, (f) 1,276 ppm, (g) 2,775 ppm of emulsifier, (h) $0.4 \mathrm{~mm}$ solid plastic spheres for comparison 


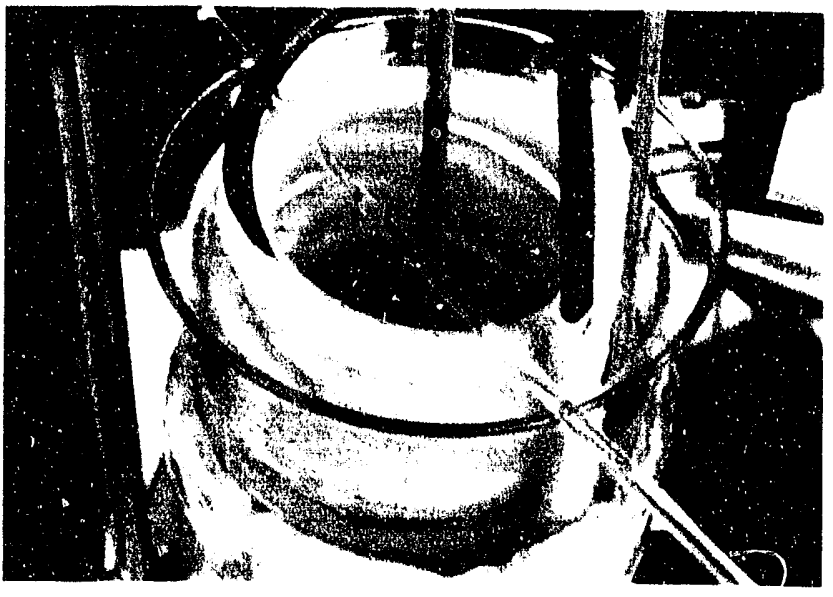

(a)

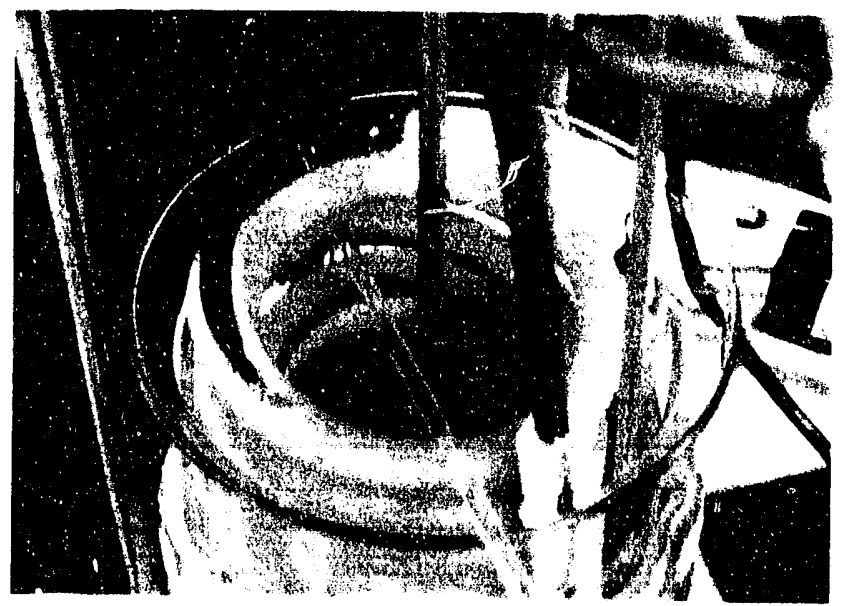

(b)

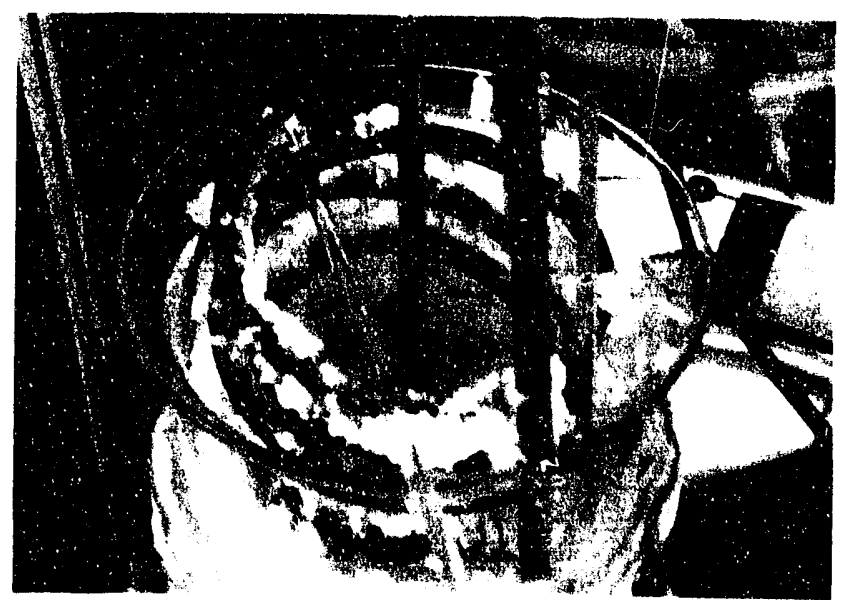

(c)

FIG 4

Photographs of solidified hexadecane produced in the simulated heat exchanger experiments with various amounts of emulsifier : (a) $0 \mathrm{ppm}$, (b) $186 \mathrm{ppm}$, (c) $625 \mathrm{ppm}$ of emulsifier 
homogeneous suspensions. The size of the particles can be controlled by the amount of the emulsifier and the degree of mixing.

The results of the simulated heat exchanger experimental system described in Fig. 2 are shown in Fig. 4. After the temperature of the mixture was lowered well below the freezing temperature of hexadecane (i.e., $<11^{\circ} \mathrm{C}$ ), the heat exchanger was pulled out of the reservoir tank. and the surface of the copper tube heat exchanger was photographed. Fig. 4(a) shows the result when no emulsifier was used. When the mixture was stirred, it became an emulsion of water and hexadecane even though there was no emulsifier present. When cold water was circulated through the heat exchanger, a thin solid layer of hexadecane coated the surface of the copper tube, and this layer continued to grow thicker. After a few minutes neither liquid nor solid hexadecane particles existed in the mixture any longer. All the hexadecane was solidified and stuck on the surface of the copper tube heat exchanger as shown in Fig. 4(a). This suggests that it is impossible to conduct a recirculating flow loop experiment of this type without using an emulsifier. The solidified hexadecane layer was stiff and difficult to scrape from the surface of the heat exchanger.

Fig. 4(b) shows the result when 186 ppm of emulsifier was used. Even though this amount of emulsifier produced fairly uniform fine hexadecane suspensions in the direct contact heat exchange system, it was not enough to produce solid hexadecane particles in the recirculating flow loop system which contained a heat exchanger. Again, all the hexadecane solidified on the surface of the heat exchanger, and there was no hexadecane left in the mixture. However, the solidified hexadecane layer was softer than the one produced without emulsifier.

Fig. 4(c) shows the result when 625 ppm of emulsifier was used. One can observe remarkable changes from those shown in Figs. 4(a) and (b). The hexadecane solidified into very small particles which did not stick to the surface of the heat exchanger. The white solid particles on the surface of the heat exchanger shown in Fig. 4(c) did not stick to the surface but rather lay there. Because the fraction of hexadecane in the mixture was $25 \%$, a thick layer of fine hexadecane formed $n$ the top of the fluid, occupying almost half of the volume of the mixture when the suspension was left without stirring. As the amount of emulsifier was increased, the size of the solidified hexadecane particles became smaller. The solidified hexadecane was too small to ve seen by the naked eye when approximately $3,000 \mathrm{ppm}$ of emulsifier was used. When that mixture was left for a long time without stirring, a thick, creamy solid hexadecane layer was formed at the top of the mixture.

\section{Cenclusions}

1. A small amount ( 60 to $2,775 \mathrm{ppm}$ ) of emulsifier produces a significant effect on the solidification of a phase-change material (i.e., hexadecane) in a mixture with water.

2. During the freezing process, the size of the solidified hexadecane particles depends on the amount of emulsifier in the water-PCM mixture. When the amount of emulsifier is increased, the size of the solidified particles becume smaller. 
3. In order to obtain uniform, stable solid particle suspensions, the amount of emulsifier should be greater than a critical value. The critical value for the case of a $25 / 75 \%$ mixture of hexadecane in water was found to be $680 \mathrm{ppm}$.

4. The emulsifier affects the interface dynamics between a heat exchanger surface and the PC.M, dictating whether the hexadecane will stick to that surface or not.

5. Mixing is an important factor that controls the size of solidified PCM particles.

\section{Acknowledgement}

The authors acknowledge the financial support of the U.S.Department of Energy, Office of Buildings and Community Systems, under its Grant No. DE-FG01-90CE26602.

\section{References}

1. P.J.Winters and R.J.Kooy, "Direct freezing ice slurry district cooling system evaluation," International District Heating and Cooling Association, 82nd Annual Conference (1991).

2. C.Cleary, S.Day, R.Lindsay, C.Murray, R.Gupta, B.Larkin, H.Thompson, M.Wiggin, and J.C.O'C.Young, "Hydraulic characteristics of ice slurry and chilled water flows," IEA District Heating : Advanced Energy Transmission Fluids - Final Report of Research (1990).

3. Y.I.Cho, E.Choi, and H.G.Lorsch, "A novel concept for heat transfer fluids used in district cooling systems", ASHRAE Transactions, Vol.97, Pt.2 (1991.).

4. P.Charunyakorn, S.Sengupta, and S.K.Roy, "Forced convection heat transfer in microencapsulated phase change material slurries: flow in circular ducts," Int. J. Heat \& Mass Transfer, Vol.34, No.3, pp 819-833 (1991). 


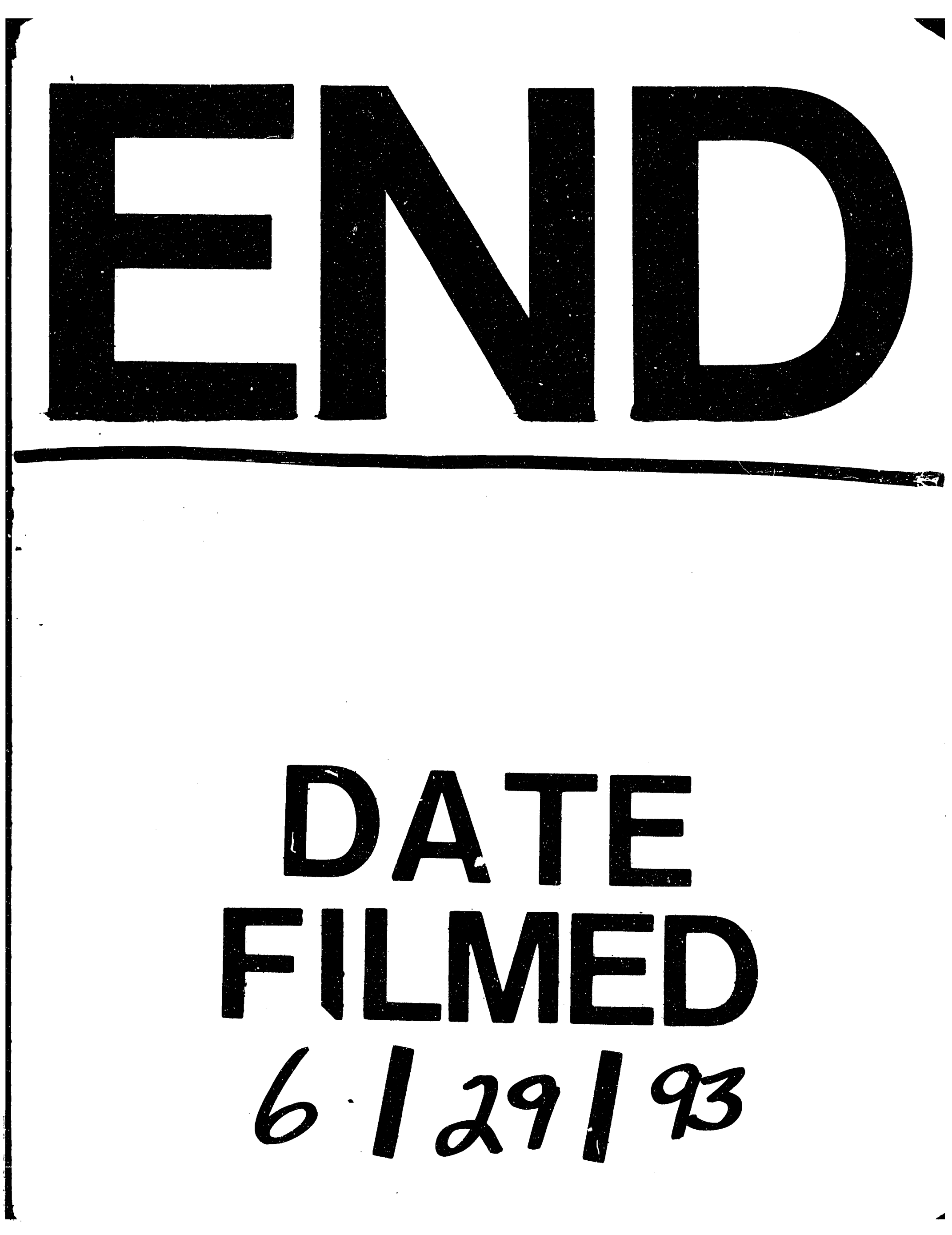


